CORPUS $\begin{aligned} & \text { Corpus } \\ & 4 \mid 2005 \\ & \text { Les corpus politiques : objet, méthode et contenu }\end{aligned}$

\title{
Le sens du texte : entre opacité statistique et transparence énonciative
}

Dominique Desmarchelier

\section{OpenEdition}

1 Journals

Édition électronique

URL : http://journals.openedition.org/corpus/372

DOI : $10.4000 /$ corpus.372

ISSN : 1765-3126

Éditeur

Bases; corpus et langage - UMR 6039

Édition imprimée

Date de publication : 1 décembre 2005

ISSN : 1638-9808

Référence électronique

Dominique Desmarchelier, "Le sens du texte : entre opacité statistique et transparence énonciative », Corpus [En ligne], 4 | 2005, mis en ligne le 01 septembre 2006, consulté le 08 septembre 2020. URL :

http://journals.openedition.org/corpus/372 ; DOI : https://doi.org/10.4000/corpus.372

Ce document a été généré automatiquement le 8 septembre 2020

(c) Tous droits réservés 


\title{
Le sens du texte : entre opacité statistique et transparence énonciative
}

\author{
Dominique Desmarchelier
}

Introduction

1 L'analyse du discours, dans une perspective argumentative, lorsqu'elle s'applique à des corpus qu'on qualifiera de «lourds", place le linguiste face à des choix méthodologiques parfois délicats : comment concilier le désir de prendre en compte la globalité du texte (de 200000 à plus d'un million d'occurrences), ce que permet effectivement un traitement lexicométrique, et le souci de mettre en évidence les orientations énonciatives et argumentatives, qui se fonderont sur des hypothèses sémantiques préexistant au corpus? Comment passer d'un traitement statistique opaque par essence, puisqu'il est capable de saisir en aveugle, de classer, de trier toutes les unités présentes dans le corpus, à l'analyse fine de l'émergence d'arguments reposant non seulement sur le jeu de l'interaction, sur la présence de connecteurs ou d'opérateurs (mais, parce que, justement, décidément, finalement...), de modalisations (pouvoir, devoir, vouloir, savoir, croire...), mais aussi sur les différentes formes d'implicites sémantiques ou pragmatiques. Comment, enfin, faire en sorte que les hypothèses sémantiques puissent orienter la collecte des données statistiques et que les résultats fournis par la lexicométrie éclairent le regard du sémanticien?

2 J'illustrerai mes propos à partir d'une étude collective ${ }^{1}$ portant sur l'« Argumentation dans l'espace public contemporain, le cas du débat sur l'immigration». Les corpus recueillis à cette occasion portaient sur les débats parlementaires, les débats télévisés, les textes officiels relatifs à la scolarisation d'enfants issus de l'immigration, les articles de l'Encyclopcedia Universalis, des sites Internet, des discussions entre étudiants et des conversations chez un marchand de journaux. Cette recherche exploite les compétences de deux équipes en analyse argumentative d'une part, et en analyse du discours et lexicométrie d'autre part. 
1. Quel type d'objet pour l'analyse argumentative ? La conception de l'argumentation conditionne le choix du corpus

On peut considérer qu'il existe au moins trois conceptions de l'argumentation :

-Argumentation $1:$ «Au moyen de...»

"Moyen de persuader un auditoire de la justesse d'une thèse ». On parle de " techniques discursives permettant d'accroître l'adhésion des esprits aux thèses qu'on présente à leur assentiment » [Perelman \& Olbrechts-Tyteca 1970]. Ce que certains ont pu appeler «idéal argumentatif»; (situations dialogales / monologales, débat, conversation);

-Argumentation 2 : «A travers... »

«Mode de construction d'un discours visant à le rendre plus résistant à la contestation "; discours, et contre-discours (perspective dialogique) [Ch. Plantin 1993] ;

- Argumentation 3

Adoptant une perspective ascriptiviste de la langue, Ducrot \& Anscombre ([Anscombre \& Ducrot 1983] puis [Anscombre 1995]) placent les orientations argumentatives à l'intérieur même de la langue. Dès lors, le recours à des corpus « authentiques » (fable de La Fontaine ou texte de Racine) ne peut avoir pour seule fin que de valider des hypothèses déjà étayées par des énoncés construits. Cette position les conduit à écarter de leurs recherches tout discours politique ou publicitaire.

2. Choix méthodologiques2.1 Ce que nous souhaitons prendre en compte dans

l'argumentation

- La description des interactions

7 Deux approches complémentaires seront ici en présence.

8 La première, lexicométrique, permet dans un débat de quantifier les prises de paroles, de les attribuer à différents locuteurs ou groupes, et d'opposer ainsi, dans les débats à l'Assemblée Nationale par exemple, prises de parole " autorisées » à "interruptions ». De même, dans un corpus long, il devient possible de repérer rapidement les spécificités lexicales (spécificité de l'apparition d'un évènement textuel dans telle ou telle partie d'un corpus, plutôt que dans telle autre) et les segments répétés (ensemble syntagmatique de $\mathrm{N}$ éléments apparaissant plusieurs fois dans le texte [Lafon \& Salem 1983]), en prenant en compte la dimension diachronique du corpus, afin de mettre en évidence certains glissements thématiques.

9 La seconde, s'appuyant sur des analyses argumentatives et conversationnelles, insiste davantage sur la nature des interventions et leur rôle dans la progression de l'échange. Le caractère complémentaire des méthodes utilisées apparaît clairement dès ce premier niveau que nous pourrions qualifier de descriptif.

- La catégorisation

10 Comme le montre Christian Plantin [1990], le travail de catégorisation (référentielle et non morphosyntaxique) constitue souvent la première étape vers l'élaboration d'une argumentation ${ }^{2}$. Nommer l'autre, c'est déjà le situer dans un cadre social, en l'opposant à ce qu'il n'est pas. Omnis determinatio est negatio, disait Spinoza. Cette remarque s'applique à la plupart des corpus analysés. Il n'est pas indifférent d'être classé « enfant d'immigrés ", « enfant issu de l'immigration », ou encore "élève en difficulté ». De même, la dénomination "jeunes des cités » recouvre souvent un amalgame entre 
« enfants de la deuxième génération », «jeunes ayant quitté l'école », pour ne pas dire « sauvageons ».

L'analyse lexicométrique, à travers les cooccurrences (ce qui revient à une contextualisation des termes), met également en évidence les qualifications attribuées à l'immigré : "clandestin" versus «en situation régulière", ou encore, sous forme polyphonique dans la bouche d'un député du PCF « ces étrangers qui nous dérangent ». Le recours à des formes génériques "ces gens-là ", " eux ", " tous ceux qui sont en France », permet également de ne pas spécifier avec précision les gens en question.

Nous prendrons également en compte :

- l'émergence et la circulation des arguments ;

- les marqueurs argumentatifs (connecteurs, modalisateurs) ;

- une typologie des arguments les plus récurrents : autorité, communauté, cadrage et analogie

[Breton 2001] ;

- les stratégies employées dans des lieux différents.

2.2 Choix des objets empiriques

A la différence de l'analyse conversationnelle qui a le souci de matérialiser le plus fidèlement possible dans l'espace graphique les «enjeux" de l'interaction (interruptions, chevauchements, hésitations, gestes, attitudes posturales, éléments de proxémique), l'analyse lexicométrique ne peut que balayer l'intégralité d'un " texte ", qui certes aura été enrichi (didascalies, identification des locuteurs...), mais qui présentera au final une linéarité souvent éloignée de la situation réelle de l'échange. Un travail de délinéarisation s'avèrera donc nécessaire pour redonner sa densité à l'interaction.

14 Un exemple de transformation d'un corpus brut en corpus enrichi peut-être donné :

1 Corpus brut : page du JO (édition HTML)

ENTRÉE ET SÉJOUR DES ÉTRANGERS EN FRANCE ET DROIT D'ASILE

SÉANCE 1 jeudi 4 décembre 1997

Discussion, après déclaration d'urgence, d'un projet de loi

le président. L'ordre du jour appelle la discussion, après déclaration d'urgence, $\mathrm{du}$ projet de loi relatif à l'entrée et au séjour des étrangers en France et au droit d'asile ( $n^{\circ}$ s 327, 451, 483).

Avant que la discussion ne s'engage, je ferai deux observations.

Nous avons prévu un temps assez important pour ce débat puisque le vote final interviendra mardi en huit. Je souhaite que nous disposions du temps nécessaire pour examiner le texte et les amendements,...

[1] Jean-Louis Debré, RPR. Voulez-vous que nous déposions d'autres amendements?

le président. ... sans qu'il y ait pour autant d'obstruction.

En second lieu, et surtout, comme il s'agit d'un sujet sensible, je souhaite que le débat se déroule dans le climat de dignité et de respect nécessaire.

[2] Rudy Salles, UDF. Ça dépend du Gouvernement !

le président. La parole est à $\mathrm{M}$. le ministre de l'intérieur.

[3] Jean-Pierre Chevènement, MDC, ministre de l'intérieur. Monsieur le président, mesdames, messieurs les députés, nous allons débattre de l'entrée et du séjour des étrangers et du droit d'asile. Mais parler des étrangers, c'est aussi une autre manière de parler de la France. Sur tous ces bancs, nous nous en faisons une certaine idée qui transcende souvent, et c'est heureux, les appartenances politiques.

Le peuple français, parce qu'il a mêlé historiquement des peuples appartenant au 
Nord et au Sud de l'Europe, des Flamands, des Catalans, des Bretons et des Corses, des Alémaniques et des Languedociens, ne s'est jamais défini par la souche, mais toujours par le bonheur d'être ensemble pour donner sens à son histoire.(...)

\section{Le même texte enrichi selon [Heiden 1999]}

$<$ ?NSL DDB /optb/corpus/lxp/reseda/reseda.lml.ddb 0>

$<$ DIV N=1 LEVEL=2 TITLE="ENTRÉE ET SÉJOUR DES ÉTRANGERS EN FRANCE ET DROIT D'ASILE">

$<$ TITLE>Discussion, après déclaration d'urgence, d'un projet de loi</TITLE>

$<$ SP $\mathrm{COM}=1 \mathrm{COM}=1 \mathrm{WHO}=$ 'le président' $\mathrm{PARTI}=\mathrm{NA}><\mathrm{P}>$ L'ordre du jour appelle la discussion, après déclaration d'urgence, du projet de loi relatif à l'entrée et au séjour des étrangers en France et au droit d'asile $</ \mathrm{P}><\mathrm{REM}>$ nøs 327, 451, 483</ REM>.

$<\mathrm{P}>$ Avant que la discussion ne s'engage, je ferai deux observations. $</ \mathrm{P}><\mathrm{P}><\mathrm{C}$ $\mathrm{V}=$ Nous $>$ nous $</ C>$ avons prévu un temps assez important pour ce débat puisque le vote final interviendra mardi en huit. $<\mathrm{C} V=\mathrm{Je}>\mathrm{je}</ \mathrm{C}>$ souhaite que nous disposions $\mathrm{du}$ temps nécessaire pour examiner le texte et les amendements, $\ldots</ \mathrm{P}></ \mathrm{SP}>$

$<\mathrm{SP} \quad \mathrm{COM}=2$ WHO='Jean-Louis Debré' PARTI=RPR $><\mathrm{P}>$ Voulez-vous que nous déposions d'autres amendements $?</ \mathrm{P}></ \mathrm{SP}>$

$<\mathrm{SP}$ COM=1 WHO='le président' $\mathrm{PARTI}=\mathrm{NA}><\mathrm{P}>\ldots$ sans qu'il y ait pour autant d'obstruction. $</ \mathrm{P}><\mathrm{P}>\mathrm{En}$ second lieu, et surtout, comme il s'agit d'un sujet sensible, je souhaite que le débat se déroule dans le climat de dignité et de respect nécessaire. $</ \mathrm{P}></ \mathrm{SP}>$

$<$ SP COM=2 WHO='Rudy Salles' PARTI=UDF $><\mathrm{P}>$ Ça dépend du Gouvernement $!</ \mathrm{P}><\mid$ $\mathrm{SP}>$

$<$ SP $\mathrm{COM}=1$ WHO='le président' $\mathrm{PARTI}=\mathrm{NA}><\mathrm{P}><\mathrm{C}$ V $=\mathrm{La}>\mathrm{la}</ \mathrm{C}>$ parole est à $<\mathrm{ABBR}$ EXPAN $=$ M. $>$ Monsieur $</ A B B R>$ le ministre de l'intérieur. $</ \mathrm{P}></ \mathrm{SP}>$

$<\mathrm{SP} \quad \mathrm{COM}=1$ WHO='Jean-Pierre Chevènement' PARTI=MDC TITLE="ministre de l'intérieur" $><\mathrm{P}>$ Monsieur le président, mesdames, messieurs les députés, nous allons débattre de l'entrée et du séjour des étrangers et du droit d'asile. $<C$ $\mathrm{V}=$ Mais $>$ mais $</ C>$ parler des étrangers, $c^{\prime}$ est aussi une autre manière de parler de la France. Sur tous ces bancs, nous nous en faisons une certaine idée qui transcende souvent, et c'est heureux, les appartenances politiques.</P>

$<\mathrm{P}>$ Le peuple français, parce qu'il a mêlé historiquement des peuples appartenant au Nord et au Sud de l'Europe, des Flamands, des Catalans, des Bretons et des Corses, des Alémaniques et des Languedociens, ne s'est jamais défini par la souche, mais toujours par le bonheur d'être ensemble pour donner sens à son histoire.</P>

2.3 Choix des outils

Le traitement lexicométrique facilite l'exploitation documentaire du corpus et permet son traitement statistique.

Exploitation documentaire

- Dimension du corpus et de ses partitions.

16 Sur l'ensemble du corpus, tous partis confondus, la répartition des interruptions et des interventions autorisées des orateurs est la suivante : 
Tableau 1 : Dimensions du Corpus RESEDA

\begin{tabular}{|l|c|c|c|c|}
\hline Corpus & Occurrences & Formes & Phrases & $\begin{array}{c}\text { Prises de } \\
\text { Parole }\end{array}$ \\
\hline Orateurs & 188027 & 12605 & 9776 & 2096 \\
\hline Interrupteurs & 13689 & 2423 & 2603 & $\mathbf{2 3 4 9}$ \\
\hline Total & $\mathbf{2 0 1 7 1 6}$ & $\mathbf{1 5 0 2 8}$ & $\mathbf{1 2 3 7 9}$ & $\mathbf{4 4 4 5}$ \\
\hline$\%$ interruptions & $\mathbf{6 . 8 \%}$ & $\mathbf{1 6 , 1 0 \%}$ & $\mathbf{2 1 \%}$ & $\mathbf{5 2 , 8 5 \%}$ \\
\hline
\end{tabular}

17 On notera sans surprise que les interruptions se réduisent le plus souvent à une phrase (2 603 phrases pour 2349 interruptions) alors que les orateurs autorisés emploient en moyenne 4,6 phrases par interventions (9 776 phrases pour 2096 interventions).

Soit au total 4445 interventions contenant 12379 phrases soit 2,8 phrases par prise de parole.

19 Ici, la phrase doit être comprise comme un ensemble d'éléments graphiques isolé par des signes de ponctuation forte (il n'y a pas d'étiquetage morphosyntaxique pour cette recherche).

- Concordances : présentation du contexte immédiat pour une forme donnée. L'exemple de « courage » (extraits) :

Il y a 2 occurrences de « courage » dans le corpus reseda/sp com=1-sp-parti-COM

\begin{tabular}{|c|c|c|c|}
\hline $\begin{array}{l}\frac{3(1627)}{\text { Andre }} \\
\text { Gerin, com }\end{array}$ & $\begin{array}{r}\text { une politique de } \\
\text { gauche qui réussisse } \\
c^{\prime} \text { est cela le }\end{array}$ & courage & $\begin{array}{l}\text { aujourd' hui } \\
\text { Monsieur le president } \\
\text { monsieur le }\end{array}$ \\
\hline $\begin{array}{l}\text { 6.79g9 (3579), } \\
\text { Andre } \\
\text { Gerin, CoM }\end{array}$ & $\begin{array}{r}\text { qui réussisse - c' est de } \\
\text { ce cóté qu' il faut } \\
\text { chercher le }\end{array}$ & courage & $\begin{array}{l}\text { je voudrais appelet } \\
\text { plus perticulierement } 1 \\
\text { attention }\end{array}$ \\
\hline
\end{tabular}

21 Il y a 7 occurrences de « courage » dans le corpus reseda/sp com=1-sp-parti-SOC 


\begin{tabular}{|c|c|c|c|c|}
\hline & 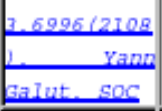 & $\begin{array}{l}\text {, sociale et culturelle } \\
\text { nous devons avoir le }\end{array}$ & courage & $\begin{array}{l}\text { fe dire qu' il n' } y \text { a pas de } \\
\text { lien direct entre } \\
\text { mmigration et }\end{array}$ \\
\hline & \begin{tabular}{|l|} 
4.6996(2816 \\
Jean- \\
Garc \\
Dyrault, SOC \\
\end{tabular} & $\begin{array}{l}\text { mes chers collègues, } \\
\text { le Gouvernement a eu } \\
\text { le }\end{array}$ & courage & $\begin{array}{l}\text { de présenter un texte } \\
\text { Equilibré, un texte } \\
\text { amendé, }\end{array}$ \\
\hline 3 & \begin{tabular}{|r|}
$5,6996(3329$ \\
Gerard \\
Gouzeg, SOC \\
\end{tabular} & $\begin{array}{r}\text { vos électeurs dans le } \\
\text { mauvais sens du poil ! } \\
\text { Ayez le }\end{array}$ & courage & $\begin{array}{l}\text { Je résister. Allez voir } \\
\text { fos électeurs et expliquez }\end{array}$ \\
\hline 4 & 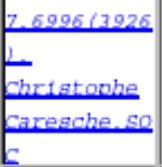 & $\begin{array}{r}\text { un hommage } \\
\text { particulier . il fallait } \\
\text { avoir le }\end{array}$ & courage & $\begin{array}{l}\text { t la volonté de dire la } \\
\text { érité aux Français, e' } \\
\text { est-a-dire que }\end{array}$ \\
\hline 5 & \begin{tabular}{|l|} 
Q.69g6/404I \\
Gerand \\
Gouzeg.soc \\
\end{tabular} & $\begin{array}{r}\text { quelle autre politique } \\
\text { alors qu' ils n' ont pas } \\
\text { eu le }\end{array}$ & courage & $\begin{array}{l}\text { Je s' exprimer ici-même, } \\
\text { se bormant à l' expliquer } \\
\text { devant }\end{array}$ \\
\hline & \begin{tabular}{|l|} 
R.6996/4045 \\
Gerand \\
Gouzeg_SoC \\
\end{tabular} & $\begin{array}{r}\text { de l' intérieur, qu' il } \\
\text { vous faut beaucoup de }\end{array}$ & courage & $\begin{array}{l}\text { pour résister aux } \\
\text { fritiques de ceux qui ne } \\
\text { proposent finalement }\end{array}$ \\
\hline & \begin{tabular}{|l|} 
9.6996(4445 \\
Jearc \\
Jarc \\
Dyrault, SOC \\
\end{tabular} & $\begin{array}{r}\text { les devoirs des } \\
\text { étrangers en France }- \text { il } \\
\text { fallait du }\end{array}$ & courage & $\begin{array}{l}\text { t de la franchise, } \\
\text { monsieur le ministre. } \\
\text { fous n' en }\end{array}$ \\
\hline
\end{tabular}

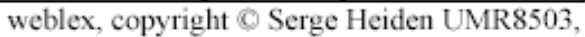
slh@ens-lsh.fr

\section{Exploitation statistique}

- Les cooccurrences

22 A l'intérieur d'un espace de rencontre préalablement défini (phrase, paragraphe), mise en évidence d'unités, à une distance $n, n+1, n+x$ d'une unité choisie. 
Cooccurrences du corpus reseda-sp-com-1-sp-parti-SOC

\begin{tabular}{|c|c|c|c|c|c|}
\hline \multicolumn{6}{|c|}{ Seuils : f $3, \mathbf{c f} 3, \mathbf{p} 5.0 \mathrm{E}-2, \mathbf{d}_{\mathbf{m}} 1000.0$} \\
\hline $\mathbf{A}$ & $\mathbf{f}_{\mathrm{A}}$ & $\mathbf{f}_{\mathrm{k}}$ & ef & $\mathbf{p}$ & $d_{m}$ \\
\hline chers collègues & 64 & 71 & 63 & $7 \mathrm{e}-108$ & 0.0 \\
\hline flux migratoires & 39 & 33 & 33 & $6 e-65$ & 0.0 \\
\hline monsieur ministre & 78 & 89 & 48 & $2 e-50$ & 1.0 \\
\hline regroupement familial & 19 & 17 & 17 & $3 e-39$ & 0.0 \\
\hline projet loi & 70 & 112 & 43 & $3 e-39$ & 1.0 \\
\hline droit asile & 102 & 72 & 40 & $10-35$ & 1.6 \\
\hline Patrick Weil & 14 & 16 & 14 & $3 e-33$ & 0.0 \\
\hline droits homme & 46 & 38 & 24 & $2 e-32$ & 2.2 \\
\hline maitrise migratoires & 15 & 33 & 15 & $2 e-28$ & 2.1 \\
\hline maîtrise flux & 15 & 39 & 15 & $4 e-27$ & 1.1 \\
\hline lois Pasqua-Debré & 64 & 19 & 18 & $6 e-27$ & 0.6 \\
\hline Monsieur ministre & 118 & 89 & 38 & le- -25 & 2.5 \\
\hline asile territorial & 72 & 17 & 17 & $1 e-25$ & 0.1 \\
\hline affaires étrangères & 10 & 12 & 10 & $7 e-25$ & 0.0 \\
\hline vie familiale & 24 & 16 & 13 & $4 e-24$ & 1.4 \\
\hline certificat hébergement & 10 & 14 & 10 & $1 e-23$ & 1.0 \\
\hline Monsieur président & 118 & 29 & 22 & $3 e-22$ & 1.1 \\
\hline motivation refus & 14 & 18 & 11 & $7 e-22$ & 2.4 \\
\hline privée familiale & 11 & 16 & 10 & $9 \mathrm{e}-22$ & 1.2 \\
\hline carte résident & 37 & 15 & 13 & $9 \mathrm{e}-22$ & 1.0 \\
\hline faveur liberté & 14 & 28 & 12 & $1 e-21$ & 2.2 \\
\hline Front national & 9 & 14 & 9 & $4 e-21$ & 0.0 \\
\hline reconduite frontière & 9 & 14 & 9 & $4 e-21$ & 2.0 \\
\hline titre sejour & 22 & 75 & 17 & $6 e-21$ & 1.1 \\
\hline
\end{tabular}

48543 couples ( 4500 paires), 1373 couples sélectionnés

(Weblex - Serge Heiden UMR8503)

- Les segments répétés

Ensembles syntagmatiques de $\mathrm{n}$ éléments contigus, les plus fréquents. Exemple dans le sous-corpus du parti socialiste :

Segments Répétés des SOC (sur l'ensemble des 8 séances) de fréquence 5, 4 et 3

“ les autorités de la République ont toujours le droit de donner asile à tout étranger persécuté en raison de son action en faveur de la liberté ou qui sollicite la protection de la France pour un autre motif" (4 occ.)

"Tout homme persécuté en raison de son action en faveur de la liberté a droit d'asile sur les territoires de la République " ( 3 occ.)

"la dépénalisation du défaut de déclaration d'entrée sur le territoire " 4 occ.)

"le relèvement d'un arrêté de reconduite à la frontière depuis " $(3 \mathrm{occ}$.)

"l'article 8 de la Convention européenne des droits de l'homme " (3 occ.)

“ loi sur l'entrée et le séjour des étrangers en France " (5 occ.)

"d'une commission du titre de séjour" (3occ.)

" des cas d'attribution de plein droit" ( 3 occ.)

"l'institution d'une carte de séjour" (3 occ.)

“ là où il n'y avait que " (3 occ.)

“la prolongation de vingt-quatre heures du délai " ( 3 occ.)

“Le préambule de la constitution de 1946 " (3 occ.)

" responsable de la montée du Front national " (3 occ.)

Segments répétés avec retour au contexte. Exemple :

$\mathrm{JO}$ : séances AN 3, 6 et 8 . Argumentaire préétabli par les députés communistes

André Gerin, COM. (séance $3: 4 / 12 / 97$ ) On prétendait travailler au rayonnement de la pensée française en commençant par fermer portes et fenêtres. Si nous pouvions nous dégager des passions du moment, nous comprendrions peut-être que ces étrangers qui nous dérangent peuvent nous rendre un grand 
service : nous inciter à nous interroger sur notre société, notre identité et notre culture.

André Gerin, COM. (séance $6: 25 / 2 / 98$ ) Comment la droite pouvait-elle prétendre travailler au rayonnement de la pensée française en commençant par fermer portes et fenêtres?

Si nous pouvions nous dégager des passions du moment, nous comprendrions peut-être que ces étrangers qui nous dérangent peuvent nous rendre un grand service en nous incitant à nous interroger sur notre société, notre identité et notre propre culture.

Analyse contrastive

- Spécificités

Il est possible de comparer la fréquence absolue d'une unité (nb. d'occurrences) dans les différentes parties du corpus, ou, plus pertinemment, d'étudier la fréquence relative des unités (fréquence absolue dans les sous-corpus/taille des sous-corpus). Mais l'outil statistique le plus performant reste les spécificités. Exemple :

Spécificités décroissantes de la partie 1 du corpus reseda-sp-com-1-sp-parti-COM

\begin{tabular}{|c|c|c|c|c|c|c|c|c|c|c|c|c|c|c|c|c|c|}
\hline construire & 15 & 9. & $8+$ & & -1 & & $1+$ & & -1 & 0 & -1 & 0] & -2 & 1 & -1 & 0 & -1 \\
\hline poltique & 436 & 58 & $7+1$ & 103 & $2+$ & 19] & $3+1$ & 123 & $2+$ & 4 & -1 & 66 & -8 & 50 & -4 & 4 & $1+$ \\
\hline voullons & 62 & 17 & $7+$ & & $\mid-1$ & 4 & $2+$ & 10 & -1 & & $1+1$ & ] & -3 & & $1+$ & 0 & -1 \\
\hline cuture & 36 & 13 & $7+$ & & -1 & & & & -1 & & -1 & 2 & -3 & 3 & .2 & 4 & $5+$ \\
\hline avec & 542 & 62 & $6+$ & 109 & !+ & 17 & $2+$ & 117 & -1 & & -1 & 117 & -2 & 112 & $1+$ & 2 & -1 \\
\hline députes & 78 & 18 & $6+$ & 9 & -2 & & $2+$ & 32. & $4+$ & & -1 & 7) & 4 & 7 & -3 & 0 & -1 \\
\hline teste & 61 & 15 & $0+$ & 10 & -1 & & -1 & 13 & -1 & & $1+$ & 11 & -1 & 11 & -1 & 0 & -1 \\
\hline tumane & 35 & 11 & $6+$ & 4 & -1 & 0 & -1 & 12. & $1+$ & & $2+$ & $\mathrm{J}$ & -2 & 2 & .2 & 0 & -1 \\
\hline combattre & 23 & 9 & $0+$ & 2 & -1 & - & $1+$ & 6 & $1+$ & & -1 & 1) & -3 & 4 & -1 & 0 & -1 \\
\hline suspicion & $\underline{\underline{2}}$ & 9 & $6+$ & 6 & $1+$ & 0 & +1 & 6 & $1+$ & & -1 & & -2 & 의 & .3 & 0 & -1 \\
\hline progressisie & 13 & 7 & $6+$ & 1 & -1 & 0 & .11 & 5] & $1+$ & & -1 & 0 & -2 & 0 & .2 & 0 & \\
\hline fermer & 8 & 6 & $6+$ & & -1 & 0 & -1 & 0 & -1 & 0 & -1 & & -1 & 1 & -1 & a & \\
\hline fraternite & 8 & 6 & $6+$ & & -1 & 0 & .1) & 0 & .11 & & -1 & & .1 & & & 0 & \\
\hline
\end{tabular}




\begin{tabular}{|c|c|c|c|c|c|c|c|c|c|c|c|c|c|c|c|c|c|}
\hline \multicolumn{2}{|c|}{ Partien: } & \multirow{2}{*}{\multicolumn{2}{|c|}{$\frac{\text { 1.Coms }}{119590}$}} & \multirow{2}{*}{\multicolumn{2}{|c|}{$\frac{2 \cdot \mathrm{SOC} r}{37377 \%}$}} & \multirow{2}{*}{\multicolumn{2}{|c|}{$\frac{\text { 3.Vertso }}{39650}$}} & \multirow{2}{*}{\multicolumn{2}{|c|}{\begin{tabular}{|c|} 
4.MD Cr \\
444140
\end{tabular}}} & \multirow{2}{*}{\multicolumn{2}{|c|}{$\frac{\text { S.RSo }}{24830}$}} & \multirow{2}{*}{\multicolumn{2}{|c|}{$\begin{array}{c}\text { 6.RPRo } \\
481980)\end{array}$}} & \multirow{2}{*}{\multicolumn{2}{|c|}{$\frac{\text { 7. UDF }}{38469 \mathrm{x}}$}} & \multirow{2}{*}{\multicolumn{2}{|c|}{$\frac{8 \cdot \mathrm{FN}^{-}}{992 \mathrm{~K}}$}} \\
\hline$T \propto$ & 1878566 & & & & & & & & & & & & & & & & \\
\hline$a$ & $\mathbf{F}$ & f & spa & fos & spd & fo & $\mathrm{sp} \mathrm{z}$ & f: & spa & f: & $\mathrm{sp}: \mathrm{x}$ & f: & sp: & f: & & & sig \\
\hline o & 0 & $\square$ & $\pi$ & $\overline{8}$ & $\pi$ & $\bar{a}$ & $\overline{8}$ & $a$ & $\pi$ & $a$ & 0 & 0 & o & $\square$ & & $\approx$ & 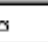 \\
\hline communistes $\alpha$ & $\underline{29}$ & 22 & $21+$ & 0 & -3 & $0:$ & $-1:$ & 2 & -2 & 0 & -1 & 2 & .2 & $3:$ & -1 & 0 & $-1 \%$ \\
\hline droited & $\underline{159}$ & 45 & $18+$ & 2]: & -2 & 11 : & $4+$ & 52 & $3+:$ & 6 & $2+$ & $11:$ & -9 & 10 & $-7:$ & 4 & $2+$ \\
\hline nous: & $\underline{1825}$ & 210 & $16+$ & 376 & $1+$ & 60: & $4 t:$ & 270 & $-21:$ & 27 & $1+$ & $469:$ & $1+$ & $\begin{array}{r}40 \\
0\end{array}$ & $2+$ & 9: & -1 \\
\hline défienced & 14: & 13 & $15+$ & $1:$ & $-1:$ & 0: & $-1:$ & 0 & $-2:$ & 0 & -1 & 0 & .2 & $0:$ & -2 & $0:$ & -1 \\
\hline populismex & 12 & 11 & $13 t$ & $1:$ & $-1:$ & $0:$ & $-1:$ & 0 & $-2:$ & 0 & -1 & 0 & .2 & $0:$ & -2 & $0:$ & -1 \\
\hline etox & 3434: & 323 & $12+$ & 747: & $3+$ & $47:$ & -4 & 770 & -2 & $5 !$ & $1+$ & 820 & -3 & $\begin{array}{r}65 \\
4\end{array}$ & -2 & 22 & $1+$ \\
\hline icéesó & $26:$ & 14 & $11+$ & 2 & -2 & $3:$ & $2+$ & 3 & $-1:$ & ! & $1+$ & 2 & .2 & $1:$ & -2 & 0 & -1 \\
\hline 1ciso & $251:$ & 45 & $10+$ & 64 & $2+$ & $8:$ & $1+$ & $33:$ & $-5:$ & 13 & $5+$ & 53: & .2 & 33 & $-3:$ & $2:$ & $1+$ \\
\hline gauchea & $\underline{121}$ & 29 & $10+$ & $\overline{17}$ & -2 & $7:$ & $2+$ & $\overline{30}$ & $1+$ & t & -1 & 17 : & -3 & 17 : & -2 & $3:$ & $2+$ \\
\hline inmigréso & $103:$ & 25 & $9+$ & $29:$ & $2+$ & 3: & $1+$ & 15 & $-2:$ & 0 & -1 & $13:$ & .4 & 16 & $-1:$ & 2 & $1+$ \\
\hline ensembleo & $\underline{75}:$ & 21 & $9+$ & $10:$ & -2 & $2:$ & $1+$ & 24 & $2+$ & 0 & -1 & 10 & .3 & $8:$ & -2 & $0:$ & -1 \\
\hline travailleta & 35 & 15 & $9+$ & 7 & -1 & $0:$ & -1 & 4 & $-2:$ & 0 & -1 & 5 & .2 & 4 & $-1:$ & $0:$ & -1 \\
\hline
\end{tabular}

2.4 Intérêt d'un traitement lexicométrique en analyse de discours

Il permet, en premier lieu de quantifier les prises de paroles dans un débat. Une partition du corpus affinera cette description par orateur, par parti, par statut institutionnel. On distinguera ainsi, orateur autorisé / interrupteur (à l'Assemblée nationale), journaliste / homme politique lors d'un débat télévisé ou radiophonique.

Ensuite, le traitement informatique, en s'appliquant à plusieurs corpus, mettra en évidence l'emploi de formes récurrentes à partir de leur fréquence relative.

Il attirera l'attention du linguiste sur certaines structures syntaxiques propres à un type de locuteurs: les interrupteurs à l'A.N ont d'avantage recours aux négations ou aux interrogations rhétoriques.

Il fait apparaître dans des corpus «lourds » des ensembles syntagmatiques récurrents (les segments répétés); l'introduction d'une perspective diachronique dans l'analyse d'un corpus peut révéler l'existence de véritables argumentaires (ex: 8 séances à l'Assemblée nationale).

29 Enfin, l'établissement d'" attirances lexicales » (ex : immigration + [clandestine, maîtrisée, galopante...]) propres à certains locuteurs (ou groupes) aboutit à une typologie des catégorisations.

30 Le croisement de ces données mettra en évidence par exemple que les interrupteurs à l'Assemblée Nationale font des phrases courtes, souvent interrogatives (réelles ou rhétoriques), sans prédicat verbal, dans le cas d'insultes ou d'invectives. On pourra ainsi éditer le « texte des interruptions".

3. Les hypothèses argumentatives pouvant guider la recherche lexicométrique

31 Si l'on fait l'hypothèse que l'émergence des arguments repose autant sur le jeu de l'interaction que sur les phénomènes de polyphonie et d'intertextualité, il sera possible d'interroger certains indices susceptibles de provoquer cette émergence.

Les outils de la deixis et les instances du discours [Benveniste 1974] je / nous / vous, aujourd'hui / hier/demain, ici / là-bas, etc. constituent les premiers indices de l'orientation énonciative du texte. Viennent ensuite les modalisations du discours (en liaison avec le temps et l'aspect). L'opposition partisane droite / gauche se traduira 
fréquemment par des échanges: «nous ne pouvons plus.../ vous n'avez pas su... ». Les diverses manifestations de la négativité discursive (négation, dénégation et connotation négative) sont également à prendre en compte. Dans un débat, ce que le camp A « fera » correspond souvent à ce que le camp B «n'a pas voulu faire ", ou a été incapable de réaliser. D'où l'importance, de pouvoir orienter la recherche automatique vers les diverses marques formelles de négation, autres que la simple locution adverbiale «ne pas » (préfixes nominaux ou adjectivaux, variantes orales, etc.).

Nous l'avons indiqué dans le début de l'article, en faisant référence aux travaux de Ducrot, la présence de connecteurs spécifiques (mais, donc, pourtant, parce que匼), bien qu'elle ne soit pas obligatoire dans un échange, est souvent l'indice de prises de positions argumentatives.

Enfin, le repérage des traces de discours rapporté conduit l'analyste à les interpréter comme autant d'appels au témoignage ou à l'argument d'autorité (pour confirmer ou réfuter une position).

Illustration du va-et-vient épistémologique entre linguistique qualitative et lexicométrie

Comme nous l'avons signalé précédemment, l'apparition de modalisations dans un texte constitue pour nous l'indice (mais non la preuve) d'un début d'argumentation. Nous avons soumis au programme Weblex un certain nombre de termes (verbes modaux mais aussi noms) appartenant à ce champ. Les résultats obtenus sur les concordances du mot « courage » en constituent une bonne illustration. Signalons que le même traitement a été appliqué à d'autres unités à valeur modale: "volonté ", « intention », « désir ».

On remarque que, si pour les composantes de la majorité au pouvoir (Communistes, Socialistes MDC, Verts) le courage relève du champ déontique (devoir, falloir), il s'inscrit au contraire sous forme de négations (ou de questions rhétoriques) chez les membres de l'opposition (RPR et UDF), associées à l'aspect accompli ou prospectif ("vous n'avez pas eu, aurez-vous?»). De même, en observant les spécificités lexicales des principaux partis, nous avons remarqué que les premiers verbes conjugués à la deuxième personne du pluriel (Vous...ez) sont respectivement «critiquez » pour les socialistes, «videz » pour le RPR et «assouplissez » pour l'UDF. A noter que ces verbes n'apparaissent dans chaque cas que dans un seul parti. Le retour au contexte met en évidence la stratégie de chaque camp. Pour la majorité, il s'agit de soutenir le texte du ministre de l'intérieur, en reprochant aux opposants de critiquer le texte, alors que pour le RPR et l'UDF, il convient de reprocher au présent texte de vider de son contenu le précédent texte (loi Pasqua-Debré), ou d'assouplir les dispositions, les rendant ainsi inapplicables.

Pour conclure

37 Rappelons l'objectif que nous nous sommes fixé. Il s'agit de réaliser un va-et-vient entre analyse lexicométrique et linguistique de l'énonciation, dans une perspective argumentative. Bien que cette démarche présente quelques difficultés méthodologiques, elle nous semble non seulement possible, mais bien plus, nécessaire face à des corpus de taille importante.

Qu'il s'agisse de débats (forme dialogale) où les opposants sont face à face (donc identifiables), de discours (monologaux) au sein desquels les arguments de "l'autre » sont actualisés sous forme d'allusions ou de discours rapporté, le recours à la 
quantification semble indispensable, en amont comme en aval, des analyses qualitatives.

En amont, parce que la lexicométrie fournit une vision "globale» du corpus, en apportant des informations non prévisibles par une simple analyse énonciative. C'est le cas notamment des segments répétés. En aval, parce qu'elle permet d'aller interroger la totalité du corpus à partir d'hypothèses argumentatives, habituellement limitées à de simples échanges locaux.

\section{BIBLIOGRAPHIE}

Anscombre J.-Cl. (1995). Théorie des Topoï. Paris : Kimé.

Anscombre J.-Cl. \& Ducrot O. (1983). L'argumentation dans la langue. Liège : Mardaga.

Benveniste É. (1974). «L'appareil formel de l'énonciation », dans Problèmes de linguistique générale. Paris : Gallimard. Tome $2: 79-88$.

Bonnafous S. \& Desmarchelier D. (1999). « Quand les députés coupent le RESEDA », Mots 60 : 93-109.

Breton Ph. $\left(2001^{2}\right)$. L'argumentation dans la communication. Paris : La découverte.

Desmarchelier D. \& Doury M. (coordinateurs) (2001). L'argumentation dans l'espace public contemporain : le cas du débat sur l'immigration. Rapport pour l'ARASH, mars 2001. GRIC (CNRS et Lyon II) et ANACOLUT (ENS Fontenay/Saint-Cloud).

Desmarchelier D. (2005). « La parole est à M. le député... », Parole \& Pouvoir 2 : 119-134.

Desmarchelier D. (2005). «La rhétorique dans les débats parlementaires », in P. Marillaud \& R. Gauthier Rhétorique des discours politiques, 25ème colloque d'Albi. Toulouse : CALS, pp. 283-296.

Ducrot O. (1993). « Les Topoï dans la 'Théorie de l'argumentation dans la langue' », in C. Plantin (éd.) Lieux communs, stéréotypes, clichés. Paris : Kimé, pp. 233-248.

Habert B. et al. (1997). Les linguistiques de corpus. Paris : Colin.

Heiden S. (1999). « Encodage uniforme et normalisé de corpus. Application à l'étude d'un débat parlementaire », Mots $60: 113-134$.

Lafon P. \& Salem A. (1983). «L'inventaire des segments répétés d'un texte », Mots $6: 161-177$.

Perelman Ch. \& Olbrechts-Tyteca L. (1970). Traité de l'argu-mentation. Bruxelles : Editions de l'Université de Bruxelles.

Plantin C. (éd.) (1990). Lieux communs, topoï, stéréotypes, clichés. Paris : Kimé. 


\section{NOTES}

1.. Desmarchelier Dominique et Doury Marianne (Coordinateurs), mars 2001 :

L'argumentation dans l'espace public contemporain le cas du débat sur l'immigration. GRIC

(CNRS et Lyon II) et ANACOLUT (ENS Fontenay/Saint-Cloud), Lyon, 413 pages.

2.. L'opération de catégorisation peut introduire une orientation argumentative, mais pas forcément. C'est un point essentiel. Des expressions comme ces gens, ces personnes... n'introduisent pas d'orientation, elles sont susceptibles d'entrer dans des prédications et des argumentations cohérentes d'orientations opposées ; elles seront dites neutres. (Rapport ADI).

\section{RÉSUMÉS}

Concilier les approches quantitatives, issues de la lexicométrie, et les analyses sémantiques et argumentatives propres à la linguistique de l'énonciation, telle est l'ambition de cet article. Plus que des résultats, on trouvera ici des pistes de dialogue entre spécialistes des « corpus lourds » et descripteurs des mouvements argumentatifs dans les textes.

From statistical opacity to enunciative transparency.

This article aims at reconciling quantitative approaches derived from lexicometry, with the semantic and argumentative analyses that are specific to enunciative linguistics. Beyond results, suggestions will be found here for a debate between specialists of "heavy corpora" and describers of argumentative movements within texts.

\section{INDEX}

Mots-clés : sémantique, argumentation, lexicométrie, catégorisation, concordances, segments répétés

\section{AUTEUR}

\section{DOMINIQUE DESMARCHELIER}

Université Paris V, CEDITEC EA 3119 